\title{
SOBRE LA NECESIDAD DEL RASURADO DE LA REGIÓN PÚBICA EN LOS PACIENTES QUE VAN A SER SOMETIDOS A CIRUGÍA UROLÓGICA ENDOSCÓPICA
}

\author{
V. MENÉNDEZ LÓPEZ, J.A. GALÁN LLOPIS, M. ELÍA LÓPEZ*, C. CARRO RUBIAS, \\ L. DE PAZ CRUZ, G. ROYO GARCÍA*, F. GARCÍA LÓPEZ
}

Servicios de Urología y *Microbiología. Hospital General Universitario de Elche. Alicante.

Actas Urol Esp. 28 (10): 761-765, 2004

\section{RESUMEN}

SOBRE LA NECESIDAD DEL RASURADO DE LA REGIÓN PÚBICA EN LOS PACIENTES QUE VAN A SER SOMETIDOS A CIRUGÍA UROLÓGICA ENDOSCÓPICA

OBJETIVO: El objetivo del estudio es determinar, mediante un ensayo clínico aleatorio controlado, si se produce algún aumento en el número de bacteriurias postoperatorias de un grupo de pacientes sometidos a cirugía urológica endoscópica, a los que no se rasura la región púbica (práctica inhabitual en la preparación quirúrgica actual).

MATERIAL Y MÉTODO: El estudio se realizó distribuyendo a los pacientes que iban a ser intervenidos mediante cirugía urológica endoscópica de forma aleatoria en dos grupos. A un grupo se le rasuró la región púbica, según las técnicas habituales, mientras que al otro grupo no se le rasuró dicha región; el resto de la preparación fue igual para ambos grupos. Se recogieron muestras de orina para su cultivo antes de la administración del antibiótico profiláctico y se volvieron a tomar a la semana de retirar la sonda vesical, ya sin tratamiento antibiótico. Se prestó especial atención a las incidencias del postoperatorio, sobre todo, a las de naturaleza infecciosa.

RESULTADOS: Se incluyeron un total de 449 pacientes, de los cuales 149 fueron retirados del estudio por diferentes causas. De estos, 149 fueron rasurados y 151 no lo fueron. En el grupo de pacientes no rasurados se observó una tasa de bacteriuria postoperatoria del 19,5\%, mientras que en el otro grupo fue del 16,6\%, no observándose diferencias estadísticamente significativas.

CONCLUSIÓN: La conclusión a la que se llegó es que no se produce un aumento de bacteriurias postoperatorias en los pacientes sometidos a cirugía urológica endoscópica, a los que no se les rasura la región púbica, en comparación con el grupo de pacientes que son rasurados con cuchillas desechables.

PALABRAS CLAVE: Rasurado. Resección transuretral. Infecciones urinarias.

\section{ABSTRACT}

ON THE NEED OF PUBIC REGION SHAVING IN PATIENTS UNDERGOING ENDOSCOPIC UROLOGIC SURGERY

OBJECTIVE: The objective of this study is to assess the effects of preoperative shaving of the pubic region on postoperative bacteriuria after endoscopic urological surgery.

MATERIALS AND METHOD: The study was carried out distributing the patients undergoing endoscopic urological surgery in a controlled randomized way in two groups. In a group the pubic region was shaved, according to the habitual techniques, while the other group was not shaved; the rest of the preparation was the same for both groups. Urine samples were collected for their culture before the administration of the prophylactic antibiotic and a week after the removal of the Foley catheter, yet without antibiotic treatment. Special attention was paid to the postoperative incidences, mainly, those of infectious nature.

RESULTS: They were included a total of 449 patients, of which 149 were removed from the study by different causes. Of these, 149 were shaved and 151 were not it. In the group of unshaved patients a 19,5\% of postoperative bacteriurias was observed, while in the other group it was of $16,6 \%$. Differences in both groups were not statistically significant.

CONCLUSIONS: We conclude that there is no an increase of postoperative bacteriurias in the unshaved patients undergoing endoscopic urological surgery, compared with the group of patients shaved with disposable bladders.

KEY WORDS: Shaving. Transurethral resection. Urinary infections. 
$\mathrm{E}^{1}$ rasurado de la piel que va a ser incidida a causa de una intervención quirúrgica ha sido aceptado como una norma internacionalmente asumida. Al indagar sobre la utilidad de esta costumbre se aducen razones como el que los pelos son una fuente de bacterias, dificultan la planificación de la incisión o impiden la fijación de los vendajes ${ }^{1}$. En 1948 Rowbotham $^{2}$ y en 1952 Oliver ${ }^{3}$ resaltaron la importancia del rasurado de la cabeza antes de proceder a la cirugía. Beck ${ }^{4}$ afirmó que los primeros cirujanos barberos no hacían referencia al rasurado de la piel, aunque debían afeitar la piel con pelo ya que daban mucha importancia a la correcta colocación y fijación de los vendajes. Unas de las primeras referencias sobre la necesidad de afeitar para conseguir la asepsia son la de Gustav Neuber, publicada en 1885, y la del "Manual of surgical asepsis" de 1895, donde se afirma que el campo quirúrgico debe ser siempre rasurado para eliminar todo el pelo 5 .

Aunque actualmente existen profesionales de distintas especialidades que no rasuran a sus enfermos, ésta sigue siendo una práctica habitual en muchos centros. No obstante, -y hasta la fecha-, las referencias a la cirugía urológica son escasas.

Si actualmente se está cuestionando la indicación del rasurado ${ }^{6,7}$, en cirugías en las que es necesario incidir la piel, es lógico cuestionarse cuál es la utilidad de rasurar a enfermos sometidos a cirugía urológica endoscópica. Por otro lado, sí parece ser que una de las razones apuntadas para rasurar a los enfermos es evitar las infecciones de las heridas, y, dado que en los pacientes sometidos a cirugía urológica endoscópica la "herida" se halla en el interior del tracto urinario, el cultivo de la orina es el mejor método para el estudio de las infecciones relacionadas con estas prácticas.

El presente estudio pretende averiguar si se produce un aumento de las infecciones urinarias postoperatorias en un grupo de pacientes a los que no se les rasura la región púbica y sometidos a cirugía urológica endoscópica.

\section{MATERIAL Y MÉTODOS}

Para la realización de este estudio se incluyeron aquellos pacientes mayores de 18 años y de ambos sexos que ingresaron para ser intervenidos de cirugía urológica endoscópica en nuestro hospital. Se excluyeron aquellos que habían estado ingresados más de 24 horas antes de la intervención o que se preveía que por alguna razón deberían permanecer ingresados más tiempo del habitual, los que habían sido rasurados en un periodo de tiempo reciente (2 semanas antes) o que se rasuraban habitualmente la zona genital, los que estaban tomando antibióticos inmediatamente antes de la cirugía o padecían enfermedades que obligaban a una pauta especifica de antibióticos (p.ej., endocarditis). Tampoco entraron en el estudio aquellos enfermos que padecían patología que podía comprometer su estado inmunitario, o aquellos enfermos que se preveía podían precisar sonda en el postoperatorio durante un periodo de tiempo superior a una semana.

Se realizó un ensayo clínico aleatorio controlado con muestreo consecutivo. La asignación de los pacientes a cada rama del estudio se realizó de forma aleatoria simple. A un grupo de pacientes se les rasuró la región púbica según la forma de proceder habitual en nuestro centro (es decir, con cuchillas desechables), mientras que al otro grupo de pacientes no se les rasuró. El resto de la preparación para la intervención fue igual en ambos grupos. Se realizó a todos los pacientes un cultivo de orina, procedente de la parte media de la micción previo lavado de los genitales externos con detergente sin antiséptico que se recogió en un recipiente estéril, al ingreso y a la semana de la retirada de la sonda. En los pacientes portadores de sonda vesical en el preoperatorio, la orina se obtuvo a través de la misma, desechando el primer chorro. Los datos clínicos y de seguimiento de los pacientes se obtuvieron de las historias clínicas y de la entrevista con el paciente en la primera visita del postoperatorio.

El urocultivo se consideró positivo si se aislaron $\geq 10^{3} \mathrm{ufc} / \mathrm{ml}$ de un microorganismo en cultivo puro ${ }^{8,9}$. En más del $80 \%$ de los casos el microorganismo se aísla en recuentos $\geq 10^{5} \mathrm{ufc} / \mathrm{ml}^{10}$. En mujeres con disuria y síndrome uretral, recuentos inferiores a $10^{3} \mathrm{ufc} / \mathrm{ml}$ pueden indicar una infección ${ }^{11}$.

En primer lugar se llevó a cabo una comparación de las características de ambos grupos de 
intervención para comprobar la homogeneidad de ambos en cuanto a posibles factores de riesgo de infección. La comparación entre dos variables cualitativas se realizó mediante el test de chi-cuadrado, y se aplicó la corrección de Yates cuando fue necesario.

\section{RESULTADOS}

Se incluyeron un total de 449 pacientes, de los cuales tuvieron que ser retirados del estudio 149 $(33,2 \%)$ por incidencias durante el mismo. Estas incidencias se desglosan en: 66 casos en los que el cultivo previo a la cirugía fue positivo, $21 \mathrm{con}$ cultivo preoperatorio contaminado, 19 por incidencias asociadas a la cirugía, 25 por no acudir a la visita postoperatoria o por no haberse realizado el cultivo de orina tras la retirada de sonda y 18 por presentar el cultivo postoperatorio contaminado. El número de pacientes que permanecieron a lo largo de todo el estudio fue de 300.

Para la comparación entre los grupos se recogieron las siguientes variables: sexo, edad, cirugía endoscópica urológica previa, infecciones urinarias previas y si llevaban sonda uretral antes de la cirugía (Tabla 1). El tipo de cirugía predominante fue el de las resecciones de tumores vesicales y de próstata (Tabla 2 ).

En 54 casos el cultivo postoperatorio fue positivo, siendo el germen aislado en 34 de ellos resistente al antibiótico utilizado profilácticamente para la intervención quirúrgica. De estos 54

\section{Tabla 1}

Relación del rasurado con varias variables clínicas

\begin{tabular}{|c|c|c|c|}
\hline & No rasurado (\%) & Rasurado (\%) & $\mathbf{p}$ \\
\hline \multicolumn{4}{|l|}{ Sexo } \\
\hline Hombre & $137(48,1 \%)$ & $148(51,9 \%)$ & $<0,05$ \\
\hline Mujer & $12(80,0 \%)$ & $3(20,0 \%)$ & \\
\hline \multicolumn{4}{|c|}{ Cirugía endoscópica previa } \\
\hline No & $101(50,8 \%)$ & $98(49,2 \%)$ & NS \\
\hline Sí & $48(47,5 \%)$ & $53(52,5 \%)$ & \\
\hline \multicolumn{4}{|c|}{ Infecciones urinarias previas } \\
\hline No & $124(47,9 \%)$ & $135(52,1 \%)$ & NS \\
\hline Sí & $25(61,0 \%)$ & $16(39,0 \%)$ & \\
\hline \multicolumn{4}{|c|}{ Portador sonda vesical } \\
\hline No & $144(50,0 \%)$ & $144(50,0 \%)$ & NS \\
\hline Sí & $5(41,7 \%)$ & 7 (58,3\%) & \\
\hline \multicolumn{4}{|c|}{ Edad (media \pm DS) } \\
\hline & $65,5 \pm 9,8$ & $66,0 \pm 10,4$ & NS \\
\hline
\end{tabular}

Tabla 2

Procedimientos quirúrgicos

\begin{tabular}{lc}
\hline $\begin{array}{l}\text { Procedimientos } \\
\text { quirúrgicos }\end{array}$ & $\begin{array}{c}\text { No de } \\
\text { procedimientos (\%) }\end{array}$ \\
\hline $\begin{array}{l}\text { Tumor vesical (TV) } \\
\text { Resección transuretral } \\
\text { de la próstata (RTU) }\end{array}$ & $147(49,0 \%)$ \\
RTU + litiasis vesical & $96(32,0 \%)$ \\
TV + RTU & $15(5,0 \%)$ \\
Estenosis de la uretra & $9(3,0 \%)$ \\
Litiasis vesical & $2(0,7 \%)$ \\
Otros procedimientos & $10(3,3 \%)$ \\
\hline
\end{tabular}

pacientes con infección de orina detectada a la semana de retirar la sonda, a 25 se les había sometido al rasurado de la zona genital y a los otros 29 no (Tabla 3). De los 6 casos registrados de septicemia postoperatoria (fiebre mayor de $38,5 \stackrel{\circ}{\circ}$, escalofríos y hemocultivos positivos), 3 tenían el cultivo de orina postoperatorio positivo y en 5 de ellos se había procedido al rasurado (Tabla 4).

\section{DISCUSIÓN}

En la piel existen dos tipos de bacterias catalogadas como residentes y transeúntes ${ }^{12}$. Las primeras no suelen ser patógenas, mientras que las transeúntes, que se suelen hallar en el pelo, son en el $90 \%$ de los casos estafilococos coagulasa negativa. Las residentes son fundamentalmente gram positivos (Corynebacterium sp y Propionibacterium sp) ${ }^{13}$.

Tabla 3

Rasurado y cultivos postoperatorios

\begin{tabular}{lccc}
\hline & $\begin{array}{c}\text { Cultivo } \\
\text { negativo (\%) }\end{array}$ & $\begin{array}{c}\text { Cultivo } \\
\text { positivo (\%) }\end{array}$ & $\mathbf{p}$ \\
\hline Grupo de pacientes & & & \\
Rasurados & $126(83,4 \%)$ & $25(16,6 \%)$ & NS \\
No rasurados & $120(80,5 \%)$ & $29(19,5 \%)$ & \\
\hline
\end{tabular}

Tabla 4

Incidencia de septicemia postoperatoria en ambos grupos

\begin{tabular}{lccc}
\hline & No rasurado (\%) & Rasurado (\%) & p \\
\hline Septicemia & & & \\
$\mathrm{No}$ & $150(99,34 \%)$ & $144(96,64 \%)$ & NS \\
$\mathrm{Si}$ & $1(0,66 \%)$ & $5(3,36 \%)$ & \\
\hline
\end{tabular}


La preparación de la piel pretende minimizar la densidad de bacterias transeúntes que, por otro lado, no se ha visto que sean más abundantes en personas con mayor densidad de pelo. La flora bacteriana del pelo no se ve alterada por los antibióticos ni por la duración de la hospitalización ${ }^{14}$, aunque sin embargo, el $20 \%$ de dichas bacterias no se ven afectadas por los antisépticos o los lavados de la preparación quirúrgica. Estas bacterias se hallan en las "bocas" de los folículos pilosos o protegidas por el estrato córneo ${ }^{15}$. El pelo no es en sí un santuario para estos gérmenes, y es más, mediante mecanismos electroestáticos atraen partículas y gérmenes protegiendo de esa forma a la piel. Por otro lado, al cortarlo no mejoramos la limpieza de la piel subyacente; al contrario, lo que sí demostró Hamilton ${ }^{16}$ experimentalmente, es que el afeitado produce microcortes invisibles donde se alojan las bacterias transeúntes e infectan la herida. Esto sucede en el 3,1\% de los casos si se rasura en el quirófano, en el 7,1\% de los casos en los que el rasurado se realiza 24 horas antes y en el 20\% de las incisiones rasuradas más de 24 horas antes.

Uno de los primeros cirujanos más vanguardistas en cuanto a la preparación de la piel antes de la cirugía es Johnston ${ }^{17}$. Ya en 1922 empieza a preguntarse si es necesario rasurar a las pacientes que van a ser intervenidas en ginecología. El ginecólogo Stephen L. Curry nunca rasuraba el monte de venus de sus pacientes, ni ningún otro vello de la zona genital y afirmaba que sólo cuando las mujeres empezaron a cuestionar la necesidad de esta práctica se descubrió que no existían estudios prospectivos controlados que respaldaran la necesidad de la misma ${ }^{18}$. Sobre los años cincuenta empezaron a oírse voces que ponían en duda la idoneidad de dichas prácticas. Se fueron alzando voces a favor de no rasurar la zona a incidir, o en el caso de las prácticas neuroquirúrgicas, de disminuir la zona rasurada.

Cruse $^{19}$ analizó 62.939 heridas de las cuales 42.054 se consideraron limpias y encontró que en el $2,5 \%$ de las rasuradas con maquinilla se infectaron frente al $1,4 \%$ si se rasuraban con máquina eléctrica o el 0,9 si no se rasuraban. Estos resultados fueron confirmados posterior- mente por $\mathrm{Mead}^{20}$ en un estudio realizado con 8.474 pacientes intervenidos quirúrgicamente por varios especialistas distintos. Similares resultados presentaron Seropian ${ }^{21}$, Alexander ${ }^{22}$ y Balthazar ${ }^{23}$ en cirugía general, así como Adeleye $^{24}$, Olson ${ }^{25}$ y Masterson ${ }^{13}$ en ginecología. Este último sólo justifica el rasurado si interfiere mecánicamente con una aproximación precisa de la herida.

Winston ${ }^{26}$ publicó en 1992 un metanálisis en el que analizaba el papel del rasurado y la aparición de infecciones en las heridas en varios tipos de intervenciones: observó que el acto de afeitar aumenta el número de infecciones en casi toda la bibliografía. Afirmó que lo único que podría justificar el rasurado sería acelerar el tiempo de cierre de la herida, la mejor adherencia de los apósitos, o mantener el ritual (sobre todo en neurocirugía) de cara al enfermo y a la sociedad. Concluyó que no existía ninguna teoría bacteriológica ni epidemiológica, que, basada en datos empíricos, demostrase que el rasurado del pelo disminuyera la contaminación bacteriana de las heridas en neurocirugía. Scherpereel ${ }^{27}$ analizó su experiencia sobre 4.655 intervenciones neuroquirúrgicas y observó una disminución del 0,65 al 0,4\% de las infecciones quirúrgicas graves como la osteítis, y llegó a llevar ya en el año 1993 veinte años sin rasurar a sus enfermos.

En cirugía urológica reconstructiva $\mathrm{McCray}^{28}$, en un pequeño estudio sobre 70 pacientes, encontró que uno de los factores relacionados de forma significativa, desde el punto de vista estadístico, con la infección es el rasurado. Schmidbauer ${ }^{29}$ coincide con la necesidad de no rasurar en cirugía urológica, aunque no endoscópica, debido a que ello disminuye la tasa de infecciones.

En cuanto al rasurado en la cirugía urológica endoscópica sólo hemos encontrado una referencia en la bibliografía. Se trata de un trabajo publicado en 1978 por Fraser $^{30}$, en el que realizó un ensayo clínico con 100 enfermos, unos rasurados y otros no analizando la incidencia de infecciones en el postoperatorio, no encontrando diferencias entre ambos grupos en cuanto a la incidencia de infecciones por lo que desaconseja el rasurado rutinario de los enfermos. 
Nuestro grupo llevó a cabo en el año 2001 una encuesta realizada en el ámbito del XXXV Congreso de la Asociación de Urología de la Comunidad Valenciana y de la Asociación Murciana de Urología, en la cual pudimos constatar que el 52,3\% de los encuestados rasuraban habitualmente a los pacientes que iban a ser sometidos a cirugía endoscópica, mientras que el 31,8\% no lo hacían de forma rutinaria ${ }^{31}$.

Nuestro estudio parece indicar que no es necesario rasurar la región púbica a los pacientes que van ser intervenidos de cirugía urológica endoscópica, dado que, por el momento, ello no produce un aumento de las bacteriurias postoperatorias ni de las septicemias. Gueda pendiente analizar otros factores que deben afectar negativamente al enfermo rasurado por rutina, como son las incomodidades que se producen física y psíquicamente al enfermo al rasurar la zona genital en una situación que, ya de por sí, resulta estresante como sucede con una intervención quirúrgica.

\section{CONCLUSIÓN}

La conclusión a la que llegamos es que no se produce un aumento de las infecciones urinarias postoperatorias en los pacientes a los que no se les rasura la región púbica y que han sido sometidos a cirugía urológica endoscópica, frente a otro grupo de pacientes que son rasurados con cuchillas desechables.

Este trabajo ha sido financiado por la Dirección General de la Agencia para la Calidad, Evaluación y Modernización de los Servicios Asistenciales (Conselleria de Sanidad de la Generalitat Valenciana) con la beca no 003/2001.

Agradecimientos: Agradecemos a todo el personal de enfermería y auxiliar del Servicio de Urología por su estrecha colaboración durante el desarrollo de todo el proyecto y a Miguel Ángel Mora por la revisión del texto.

\section{REFERENCIAS}

1. Shaving the head: reason or ritual? Lancet 1992;340(8829):1198-1199.

2. Rowbotham GF. Acute injuries of the head. Edinburgh: Livingstone, 1949:210.

3. Oliver LC. Essentials of neurosurgery. London: Lewis, 1952: 26.

4. Beck WC. Hair and asepsis and antisepsis. Surg Gynecol Obstet 1986; 163(5):479.

5. Neuber G., citado por Clemons B. Gustav Neuber's gift to medicine. $J$ Healthc Mater Manage 1985;3:70-80.
6. Kjonniksen I, Andersen BM, Sondenaa VG, Segadal L. Preoperative hair removal-a systematic literature review. AORN J 2002;75(5):928-940.

7. Mangram AJ, Horan TC, Pearson MI, Silver LC, Jarvis WR. Guideline for prevention of surgical site infection, 1999. Hospital Infection Control Practices Advisory Committee. Infect Control Hosp Epidemiol 1999;20(4):250-278.

8. Mensa J, Gatell Jm, Jimenez de Anta MT, Prats G, Dominguez-Gil A. Guía de Terapéutica Antimicrobiana. 14 $4^{\underline{a}}$ ed. Barcelona 2004. Editorial Masson.

9. Hooton TM, Stamm WE. Diagnosis and treatment of uncomplicated urinary tract infection. Infect Dis Cin North Am 1997; 11(3):551-581.

10. Kass EH. Asymptomatic infections of the urinary tract. Trans Assoc Am Physicians 1956;69:56-59.

11. Fihn SD. Acute uncomplicated urinary tract infection in women. $N$ Engl J Med 2003;349(3):259-266.

12. Montes LF, Wilborn WH. Anatomical location of normal skin flora. Arch Dermatol 1970;101(2):145-159.

13. Masterson BJ. Skin preparation. Clin Obstet Gynecol 1988;31(3):736-743.

14. Black WA, Bannerman CM, Black DA. Carriage of potentially pathogenic bacteria in the hair. Br $J$ Surg 1974;61: 735-738.

15. Selwyn S, Ellis H. Skin bacteria and skin disinfection reconsidered. $\mathrm{Br}$ Med $J$ 1972; 1(793): 136-140.

16. Hamilton HW, Hamilton KR, Lone FJ. Preoperative hair removal. Can J Surg 1977;20(3):269-271.

17. Johnston RA, Sidall RS. Is the usual method of preparing patients for delivery beneficial or necessary? Am J Obstet Gyn 1922;4:645-650.

18. Debate over preparative shaving leaves surgeons splitting hairs. Med World News 1983;24:43.

19. Cruse PJ, Foord R. The epidemiology of wound infection. A 10-year prospective study of 62,939 wounds. Surg Clin North Am 1980; 60(1):27-40

20. Mead PB, Pories SE, Hall P, Vacek PM, Davis JH Jr, Gamelli RL. Decreasing the incidence of surgical wound infections. Validation of a surveillance-notification program. Arch Surg 1986;121(4):458-461.

21. Seropian R, Reynolds BM. Wound infections after preoperative depilatory versus razor preparation. Am J Surg 1971;121(3):251-254.

22. Alexander JW, Fischer JE, Boyajian M, Palmquist J, Morris MJ. The influence of hair-removal methods on wound infections. Arch Surg 1983;118(3):347-352.

23. Balthazar ER, Colt JD, Nichols RL. Preoperative hair removal: a random prospective study of shaving versus clipping. South Med $J$ 1982;75(7):799-801.

24. Adeleye JA. Perineovulvovaginal preoperative preparation in minor gynecological surgery. Int Surg 1976;61(9):467.

25. Olson MM, Maccallum J, Mcquarrie DG. Preoperative hair removal with clippers does not increase infection rate in clean surgical wounds. Surg Gynecol Obstet 1986;162(2):181-182.

26. Winston KR. Hair and neurosurgery. Neurosurg 1992;31(2):320-329.

27. Scherpereel B. Le non rasage. Neurochirurgie 1993;39(6): 374-375.

28. Mccray E, Martone WJ, Wise RP, Culver DH. Risk factors for wound infections after genitourinary reconstructive surgery. Am J Epidemiol 1986;123(6):1026-1032.

29. Schmidbauer CP, Porpaczy P. Bacterial nosocomial infections in urology: defective wound healing. Wien Klin Wochenschr 1982;94(9):231-235.

30. Fraser I, Macpherson S, Panagakis A. Should patients be shaved prior to transurethral surgery? Br J Urol 1978; 50(2):109-110.

31. Menéndez López V, Galán Llopis JA, Lloréns Martínez FJ, Garcia López F. Resultados de la encuesta sobre el manejo de los pacientes sometidos a cirugía endoscópica urlógica. Actas Urol Esp 2002;26(5):377-378.

Dra. V. Menéndez López

Servicio de Urología

Hospital General Universitario de Elche

03203 Elche (Alicante)

(Trabajo recibido el 2 abril de 2004) 